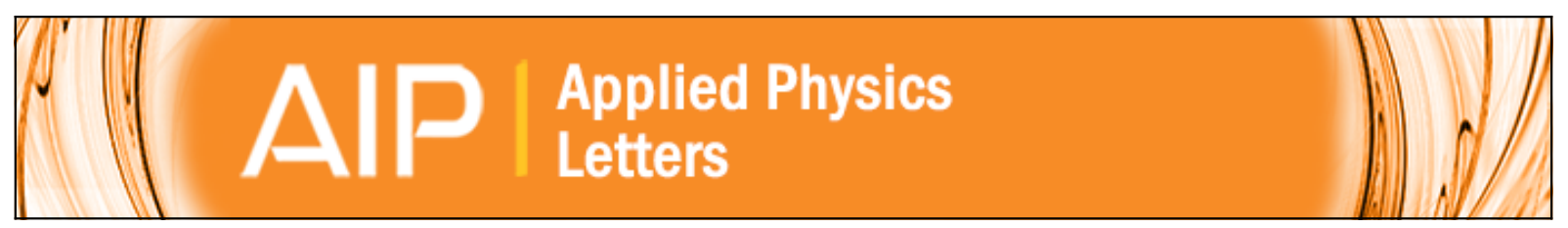

\title{
On the origin of remanence enhancement in exchange-uncoupled CoFe2O4-based composites
}
A. Quesada, F. Rubio-Marcos, J. F. Marco, F. J. Mompean, M. García-Hernández, and J. F. Fernández

Citation: Applied Physics Letters 105, 202405 (2014); doi: 10.1063/1.4902351

View online: http://dx.doi.org/10.1063/1.4902351

View Table of Contents: http://scitation.aip.org/content/aip/journal/apl/105/20?ver=pdfcov

Published by the AIP Publishing

\section{Articles you may be interested in}

Tailoring exchange coupling and phase separation in Fe-Co-Mn nanocomposites

J. Appl. Phys. 115, 17A729 (2014); 10.1063/1.4866704

Engineered spin-valve type magnetoresistance in Fe3O4-CoFe2O4 core-shell nanoparticles Appl. Phys. Lett. 103, 102406 (2013); 10.1063/1.4819956

Finite temperature performance of hard-soft composite nanomagnets and its dependence on geometry structure of composites

J. Appl. Phys. 113, 043913 (2013); 10.1063/1.4788703

A feasible approach for preparing remanence enhanced NdFeB based permanent magnetic composites J. Appl. Phys. 109, 07A710 (2011); 10.1063/1.3551744

Microstructure and exchange coupling in nanocrystalline Nd 2 ( Fe Co ) 14 B/ $\alpha$ - Fe Co particles produced by spark erosion

Appl. Phys. Lett. 86, 122507 (2005); 10.1063/1.1890474

You don't

still use this

cell phone

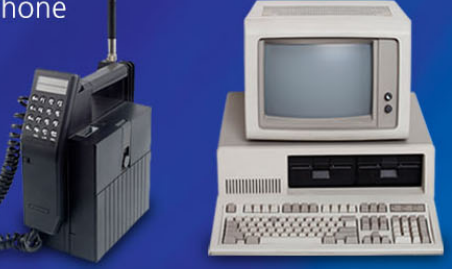

Why are you still using an AFM designed in the 80 's?
It is time to upgrade your AFM Minimum $\$ 20,000$ trade-in discount for purchases before August 31st

Asylum Research is today's technology leader in AFM 


\title{
On the origin of remanence enhancement in exchange-uncoupled $\mathrm{CoFe}_{2} \mathrm{O}_{4}$-based composites
}

\author{
A. Quesada, ${ }^{1}$ F. Rubio-Marcos, ${ }^{1}$ J. F. Marco, ${ }^{2}$ F. J. Mompean, ${ }^{3}$ M. García-Hernández, ${ }^{3}$ \\ and J. F. Fernández ${ }^{1}$ \\ ${ }^{1}$ Instituto de Cerámica y Vidrio, CSIC, 28049 Madrid, Spain \\ ${ }^{2}$ Instituto de Química-Física Rocasolano, CSIC, 28006 Madrid, Spain \\ ${ }^{3}$ Instituto de Ciencia de Materiales de Madrid, CSIC, 28049 Madrid, Spain
}

(Received 27 October 2014; accepted 10 November 2014; published online 19 November 2014)

\begin{abstract}
Abundant efforts are being devoted to the production of nanostructured composites based on ferrites where the exchange-spring mechanism yields larger energy products. However, experimental results occasionally contradict the predictions, and the origin of the phenomenology is not always thoroughly understood. Magnetic properties may eventually evolve in trends similar to that of exchange-spring magnets due to structural changes unrelated to exchange-coupling mechanisms at hard/soft interfaces. Thus, the evaluation of the possible sources of magnetic improvement is pivotal in determining and optimizing the extent of exchange-coupling in spring magnets. Here, we report the observation of remanence improvement and single-phase reversal behavior at room-temperature in $\mathrm{CoFe}_{2} \mathrm{O}_{4} / \mathrm{FeCo}$ composites, where the hard and soft phases are predominantly uncoupled. The results are explained in terms of the temperature evolution of the individual properties of $\mathrm{CoFe}_{2} \mathrm{O}_{4}$. Causes for the observation of single-phase magnetization reversal in exchange-uncoupled systems are discussed. Our results indicate that these type of phenomena must be taken into account in order to thoroughly understand the optimization required to improve the magnetic performance of $\mathrm{CoFe}_{2} \mathrm{O}_{4}$-based composites. (C) 2014 AIP Publishing LLC. [http://dx.doi.org/10.1063/1.4902351]
\end{abstract}

The improvement of permanent magnets constitutes a decades-long goal of the scientific community that has been recently refueled by risks of shortcomings in the supply of rare-earths. ${ }^{1,2}$ Several approaches to the issue have been undertaken, ${ }^{3-5}$ out of which enhancement of the properties of nanostructured ferrites through the use of the exchangespring effect has gathered large efforts. ${ }^{6-9}$ In this type of magnets, exchange-coupling at the interface between magnetically hard and soft particles leads to an increase in saturation magnetization $\left(M_{s}\right)$ and remanence $\left(M_{r}\right)$ that overcomes the loss of coercivity in yielding larger energy products. $^{10}$ Unfortunately, owing to the fact that effective exchange-spring is a quantum-mechanical effect that appears at coherent atomic interfaces in particles below a certain critical size, strict structural restrictions apply. In this framework, the use of $\mathrm{CoFe}_{2} \mathrm{O}_{4}(\mathrm{CFO})$ nanoparticles provides the opportunity of fabricating metal/ferrite hybrid compounds while avoiding the use of dissimilar starting materials. ${ }^{11}$ Partial reduction of $\mathrm{CFO}$ leads to $\mathrm{CFO} / \mathrm{FeCo}$ core-shell composites where coherent interfaces are statistically more likely than when interfaces between two different phases are engineered. In the last years, research on this class of composites has proliferated and successful exchange-coupling and magnetic improvements have been claimed. ${ }^{12-19}$ However, the physical mechanisms behind the phenomenology and the extent of effective exchange-coupling are not fully elucidated. $^{12,13,20}$ Given that $\mathrm{CFO} / \mathrm{FeCo}$ composites have been mainly studied on polycrystalline powder form, which are complex systems that can accommodate broad particles size distributions and different compositions and interfaces within the same sample, it must be kept in mind that structural and compositional variations of the individual uncoupled compounds may be responsible for phenomenology that could mistakenly be interpreted as the fingerprint of exchange-spring. In particular, CFO presents a relatively complex structure containing tetrahedral and octahedral positions. The cationic distribution and deviations from stoichiometry have a crucial influence on its magnetic properties. $^{21}$

The aim of this work is to study the magnetic properties of $\mathrm{CFO} / \mathrm{FeCo}$ nanoparticulated powders and to address effects that can give rise to $\mathrm{M}_{\mathrm{r}} / \mathrm{M}_{\mathrm{s}}$ enhancement and singlephase hysteresis curves in the absence of exchange-coupling between hard and soft phases. To this end, pure CFO samples will be studied in a first step in order to later identify the magnetic contribution of CFO in the hybrid composites. Some of our conclusions may be extrapolated to all exchange-spring systems.

CFO was synthesized following a sol-gel process using $\mathrm{Fe}$ and Co nitrates. The nitrates were dissolved in 1:2 ratio in deionized water. Citric acid was subsequently added to the solution, which was then constantly stirred at $60^{\circ} \mathrm{C}$ for $1 \mathrm{~h}$. The gel formed was dried in a hot air oven for $24 \mathrm{~h}$. The precursor obtained was calcined for $1 \mathrm{~h}$ at $400{ }^{\circ} \mathrm{C}$. The resulting powder, hereafter named "as-calcined," was subjected to two different annealing procedures: (a) annealing in air at temperatures between $500-900^{\circ} \mathrm{C}$. (b) A reduction treatment, which consisted on annealing for $1 \mathrm{~h}$ at $500^{\circ} \mathrm{C}$ under different partial pressures of a $10 \% \mathrm{H}_{2}-90 \% \mathrm{~N}_{2}$ gas mixture. The structural characterization has been done by x-ray diffraction (XRD, D8 Advance, Bruker), scanning electron microscopy, and energy dispersion spectroscopy (FE-SEM Hitachi S-4700, equipped with an EDX analysis system). Transmission electron microscopy TEM was performed 
using a JEOL 2100F transmission electron microscope (TEM/HRTEM) operating at $200 \mathrm{kV}$ and equipped with a field emission electron gun providing a point resolution of $0.19 \mathrm{~nm}$. The microscope was also coupled with an energy dispersive X-ray spectrometry (EDXS) instrument (INCA xsight, Oxford Instruments). Transmission Mössbauer spectroscopy was performed at $298 \mathrm{~K}$ using a ${ }^{57} \mathrm{Co}(\mathrm{Rh})$ source and a constant acceleration spectrometer. Isomer shifts are referred to metallic iron. For the study of the magnetic properties, we chose vibrating sample magnetometry (GMW Magnet Systems, model 3474-140 Electromagnet, LakeShore).

In order to evaluate the separate contribution of CFO to the magnetic properties of the composites, the effect of the thermal treatment in pure CFO samples is first studied. The structural characterization confirms the formation of $\mathrm{CFO}$ after the calcination process at $400^{\circ} \mathrm{C}$. Fig. 1(a) shows a series of TEM images. Particles with an average diameter of $35 \mathrm{~nm}$ are arranged in agglomerates-often larger than $1 \mu \mathrm{m}$. The interfringe distance value of $4.909 \AA$ corresponds to the
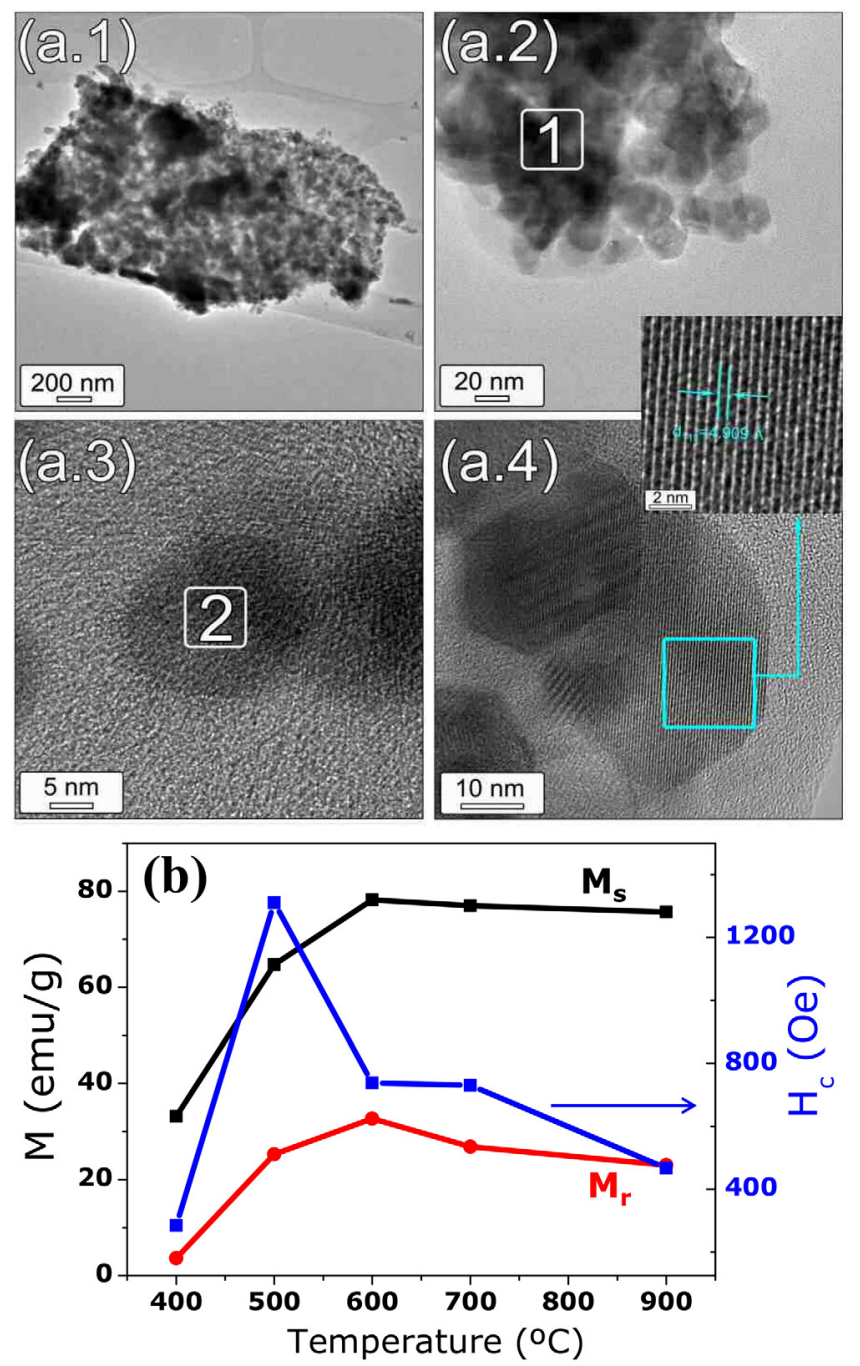

FIG. 1. (a.1-a.4) Series of HRTEM images at different magnifications corresponding to the as-calcined $\left(400^{\circ} \mathrm{C}\right)$ sample. EDS analysis was performed at regions labeled as 1 and 2 on (a.2) and (a.3). Inset of the panel (a.4) shows interplanar distances obtained from reconstructed images. (b) Saturation magnetization $\left(M_{s}\right.$, black $)$, remanence $\left(M_{r}\right.$, red) and coercive field $\left(H_{c}\right.$, blue $)$ at RT of the calcined CFO powder as a function of annealing temperature.
(111) planes of CFO. EDXS analysis reveals that the local composition is not completely homogeneous and fluctuates between individual particles, with $\mathrm{Fe}$ :Co ratios as high as 2.7:1 detected in the isolated particle labeled 2. An average compositional analysis was performed on a larger region labeled 1. An Fe:Co ratio of 1.9 was measured. Besides the specific synthesis conditions, the relatively low calcination temperature is a likely reason for the compositional variability, given that temperatures above $900{ }^{\circ} \mathrm{C}$ are reported to be employed in sol-gel synthesized CFO. ${ }^{22}$ As expected, increasing re-annealing at higher temperatures leads to larger particle sizes and homogeneity. From TEM images (not shown), we measure, at $500^{\circ} \mathrm{C}, 700{ }^{\circ} \mathrm{C}$, and $900^{\circ} \mathrm{C}$, average particle sizes of $68 \mathrm{~nm}, 80 \mathrm{~nm}$, and $95 \mathrm{~nm}$, respectively. The larger Fe:Co ratio detected for $900{ }^{\circ} \mathrm{C}$ is 2.3:1.

Magnetization curves were measured at roomtemperature (RT) for all annealing temperatures. Figure 1(b) presents the evolution of the main magnetic parameters as a function of temperature, the $400{ }^{\circ} \mathrm{C}$ data corresponding to the as-calcined sample. Focusing on the re-annealed materials, the average $M_{s}, M_{r}$, and coercivity values are $74 \mathrm{emu} / \mathrm{g}$, $27 \mathrm{emu} / \mathrm{g}$, and $\mathrm{H}_{\mathrm{c}}=812 \mathrm{Oe}$. As a function of the annealing temperature, variations in $\mathbf{M}_{\mathrm{s}}, \mathbf{M}_{\mathrm{r}}$, and coercivity of $20 \%$, $30 \%$, and $64 \%$ are, respectively, observed.

Figure 2 presents the Mössbauer spectra corresponding to the samples re-annealed at 400,700 , and $900^{\circ} \mathrm{C}$. At $400{ }^{\circ} \mathrm{C}$, the spectra are typical of a sample with a broad particle size distribution, ranging from dimensions below the superparamagnetic limit that gives rise to the central doublet, to highly crystalline larger particles that yield the modes associated with CFO. The presence of superparamagnetic particles with blocking temperatures $\left(\mathrm{T}_{\mathrm{b}}\right)$ below RT explains the low coercivity values found for the as-calcined sample. At $500{ }^{\circ} \mathrm{C}$, a maximum in coercivity is observed. The further decrease with temperature between $500-900{ }^{\circ} \mathrm{C}$ can be attributed to the increase in particle size observed by TEM. $^{23,24}$ Annealing at higher temperatures leads to larger particles and narrower size distributions as the corresponding Mössbauer spectra confirm.

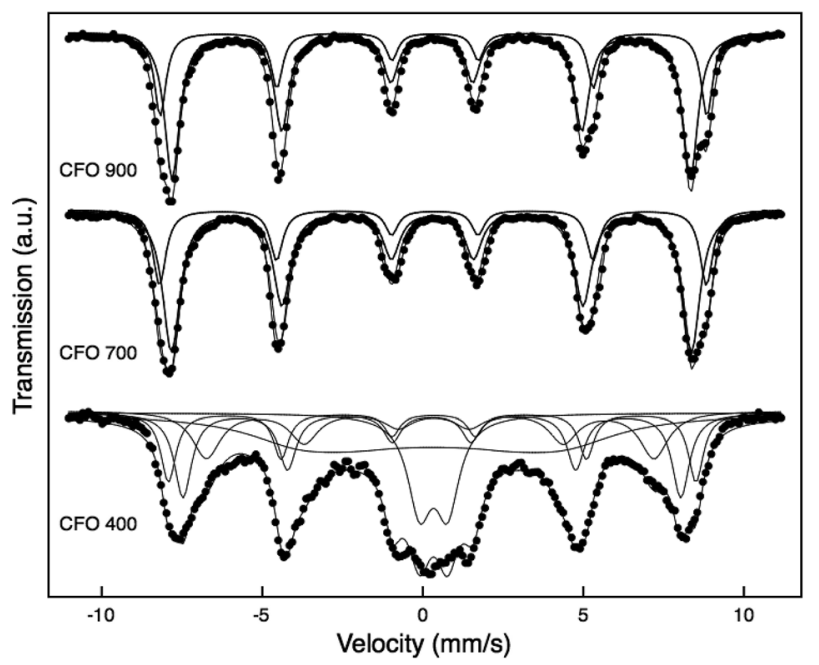

FIG. 2. Room temperature Mössbauer spectra recorded from the samples reannealed in air (bottom to top) at 400,700 , and $900^{\circ} \mathrm{C}$. 
For $700{ }^{\circ} \mathrm{C}$ and $900{ }^{\circ} \mathrm{C}$, well defined magnetic components attributed to $\mathrm{CFO}$ are observed. The spectra are composed by two sextets, one attributed to tetrahedrally coordinated $\mathrm{Fe}^{3+}\left(\delta=0.28 \mathrm{mms}^{-1}, 2 \varepsilon=-0.03 \mathrm{mms}^{-1}\right.$, and $\mathrm{H}=50.1 \mathrm{~T})$ and other corresponding to octahedrally coordinated $\mathrm{Fe}^{3+}\left(\delta=0.36 \mathrm{mms}^{-1}, 2 \varepsilon=-0.06 \mathrm{mms}^{-1}\right.$, and $\mathrm{H}=52.8 \mathrm{~T})$. However, the tetrahedral/octahedral ratio shows some changes with the preparation temperature: 0.39 and 0.45 for $700{ }^{\circ} \mathrm{C}$ and $900^{\circ} \mathrm{C}$, respectively. Variations in the cationic distribution are known to strongly influence the magnetic characteristics of $\mathrm{CFO},{ }^{21}$ and are likely the cause for the changes in remanence and saturation observed between 700 and $900^{\circ} \mathrm{C}$. Thus, the combination of TEM and Mössbauer suggests that temperature dependence of particle size distribution, local composition, and cationic distribution leads to relatively important variations in $\mathrm{M}_{\mathrm{r}}, \mathrm{M}_{\mathrm{s}}$, and coercivity. These effects have been previously reported in CFO, and the macroscopic magnetic properties arise as a combination of all of them. However, in order to later understand the phenomenology associated to the composites, it is important to at least partially quantify their influence: From our data, we calculate variations in $\mathrm{Fe}^{3+}$ tetrahedral/octahedral ratio of $14 \%$ and variations in particle size of $33 \%$, which-in combination with the variations in local composition-yield changes in remanence and coercivity of $30 \%$ and $64 \%$, respectively. Unfortunately, full quantitative analysis remains elusive since it is extremely difficult to quantify the variations in local composition and to isolate the effect on the magnetism of each individual contribution-particle size, composition, and cationic distribution.

To fabricate hybrid $\mathrm{CFO} / \mathrm{FeCo}$ composites, the ascalcined powders were reduced at $500^{\circ} \mathrm{C}$, for $1 \mathrm{~h}$, under different $\mathrm{H}_{2}$ partial pressures ranging from 1 to 20 millibar. Larger partial pressures correspond to a higher reducing strength of the thermal treatment. Figure 3 shows the associated XRD patterns. For 1 millibar, two phases are observed:

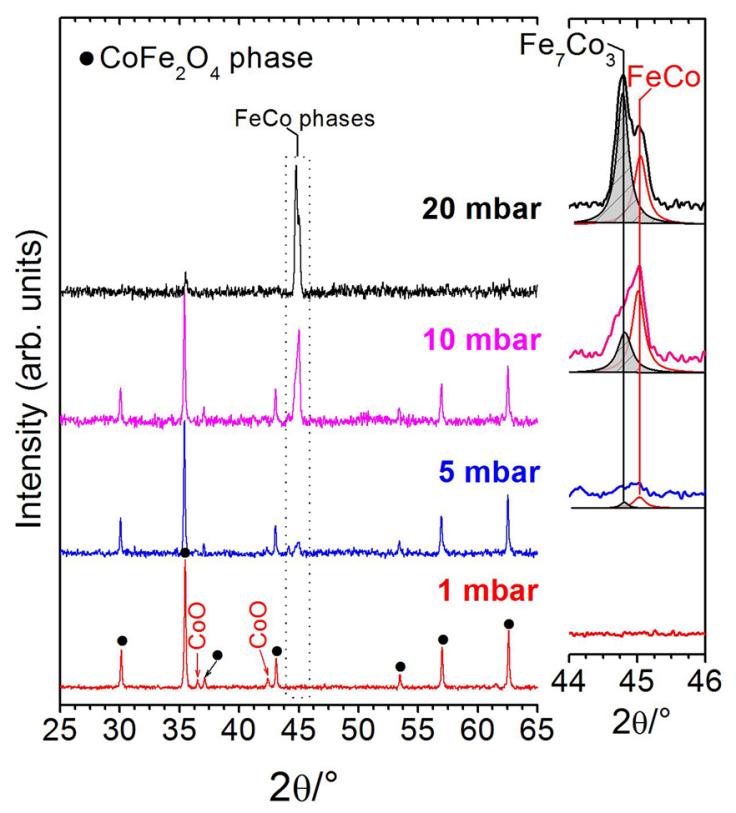

FIG. 3. XRD patterns from the powders annealed under 4 different $\mathrm{H}_{2} / \mathrm{N}_{2}$ partial pressures. Right panel shows detail of the FeCo maxima deconvolution.
$\mathrm{CFO}$ and $\mathrm{CoO}$. The formation of $\mathrm{CoO}$ suggests that the remaining $\mathrm{CFO}$ is off-stoichiometric and Co-deficient. No metallic phase is detected up to the instrument resolution $(2 \%)$. At and above 5 millibar, the diffraction maximum from $\mathrm{FeCo}$ becomes increasingly intense, indicating the progressive formation of the metallic alloy. Interestingly, two FeCo phases can be identified by deconvoluting the aforementioned peak: $\mathrm{Fe}_{65} \mathrm{Co}_{35}$ and $\mathrm{Fe}_{50} \mathrm{Co}_{50}$. From the evolution of the deconvoluted area with the reducing strength, we can infer that the reduction initially yields $\mathrm{Fe}_{50} \mathrm{Co}_{50}$ particles. As it progresses towards a complete and homogeneous reduction, the $\mathrm{Fe}_{65} \mathrm{Co}_{35}$ phase, which corresponds to the stoichiometry of the precursor CFO particles, proliferates. Thus, the composition of both hard and soft phases varies as a function of the partial pressure, indicating that the reduction favors the coexistence of metallic and oxide species with different Fe and Co contents. Furthermore, using Scherrer's formula, the crystallite sizes for the CFO phase have been obtained for the different partial pressures. The crystallite size values remain relatively constant ranging between $64-80 \mathrm{~nm}$, in agreement with the particle size obtained for the sample annealed at $500^{\circ} \mathrm{C}$ in air.

Magnetometry experiments were performed. The magnetization curves at RT corresponding to the samples annealed at $500{ }^{\circ} \mathrm{C}$ in air and under 1 and 10 millibar of $\mathrm{H}_{2} / \mathrm{N}_{2}$ are portrayed in Fig. 4(a). Taking the sample annealed in air as a reference, it is observed that after the reduction under 1 millibar, $\mathrm{M}_{\mathrm{s}}$ and $\mathrm{M}_{\mathrm{r}}$ increase to $76 \mathrm{emu} / \mathrm{g}$ and $33 \mathrm{emu} / \mathrm{g}$, respectively, while coercivity decreases from 1311 Oe to $997 \mathrm{Oe}$, which means a $32 \%$ increase in $\mathrm{M}_{\mathrm{r}}$ and a $24 \%$ decay in $\mathrm{H}_{\mathrm{c}}$.

Even though such $M_{r}$ and $M_{s}$ enhancement is typical of exchange-spring systems, the structural characterization confirmed the absence of a metallic soft phase in the 1-millibar sample. In fact, $\mathrm{CoO}$ forms under such conditions yielding a Co-deficient CFO. In addition, and considering all samples under study, the existence of two FeCo phases after partial reduction hints at local variations in composition on the remaining unreduced CFO particles. In fact, EDXS analysis on the 1-millibar sample revealed the presence of particles with $\mathrm{Fe}$ :Co ratios of 3:1, indicating large compositional fluctuations. Mössbauer spectra were recorded for the sample annealed at $500^{\circ} \mathrm{C}$ in 10 millibar of $\mathrm{H}_{2} \mathrm{~N}_{2}$ (not shown), unfortunately, the prominent formation of metallic phase prevents an accurate determination of the $\mathrm{Fe}^{3+}$ tetrahedral/ octahedral ratio. In any case, it has been reported that in CFO, reducing the Co content affects $\mathrm{M}_{\mathrm{r}}$, and we have confirmed experimentally that variations of the order of $14 \%$ in the tetrahedral/octahedral ratio may easily lead to $30 \%$ enhancements in $\mathrm{M}_{\mathrm{r}}{ }^{21}$ We stress that these mechanisms are at the origin of the magnetization improvement observed for the 1 millibar sample. For 10 millibar, the formation of metallic soft phase explains the large decrease in $\mathrm{H}_{\mathrm{c}}$.

From the $\mathrm{M}_{\mathrm{s}}$ of the reference compounds (CFO and $\mathrm{FeCo}$, an estimation of the hard:soft phase ratio can be obtained. The samples partially reduced at 5 and 10 millibar correspond to the compositions $95 \% \mathrm{CFO}-5 \% \mathrm{FeCo}$ and $53 \% \mathrm{CFO}-47 \% \mathrm{FeCo}$, respectively. They show a further increase in $\mathrm{M}_{\mathrm{s}}$ reaching $144 \mathrm{emu} / \mathrm{g}$, as is expected taking into account the substantial formation of soft FeCo. The coercive field diminishes to $263 \mathrm{Oe}$ and $\mathrm{M}_{\mathrm{r}}$ decays to $23 \mathrm{emu} / \mathrm{g}$ for 
(a)

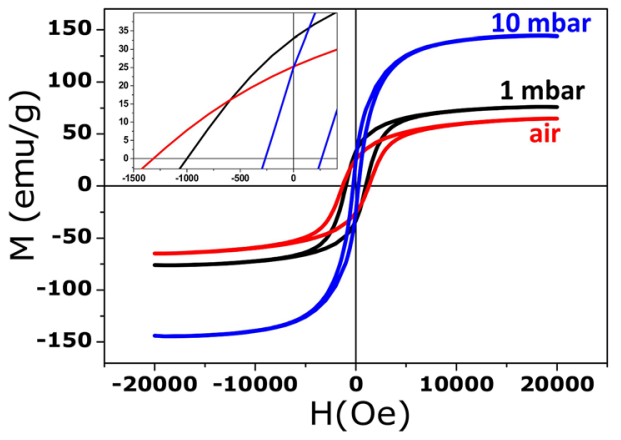

(b)

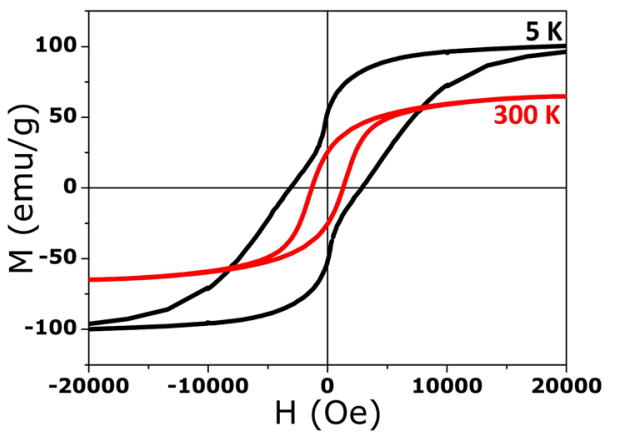

FIG. 4. (a) Magnetization curves at RT for the samples annealed at $500^{\circ} \mathrm{C}$ in air, in 1 millibar and in 10 millibar of $\mathrm{H}_{2} \mathrm{~N}_{2}$. (b) Magnetization curves at RT and $5 \mathrm{~K}$ corresponding to the sample annealed in 5 millibar of $\mathrm{H}_{2} \mathrm{~N}_{2}$.
10 millibar. The decrease in $\mathrm{M}_{\mathrm{r}}$ and the collapse of coercivity for these two composites with respect to the reference CFO suggest that the hard CFO and soft FeCo particles are not effectively exchange-coupled. Nevertheless, the hysteresis loop at RT appears to correspond to a single magnetic phase, with irreversible magnetization reversal taking place at the same nucleation field for both the hard and soft phases.

To understand this behavior, magnetization curves at low temperature were measured. Fig. 4(b) shows the hysteresis loops at $5 \mathrm{~K}$ and RT from the 5-millibar sample, where soft and hard particles coexist. Single-phase behavior is observed at RT, while a two-step reversal appears at $5 \mathrm{~K}$. Identical behavior was observed in the 1 and 10 millibar samples. Given the complexity and heterogeneity of the system, it is likely that the distribution of reversal fields of hard and soft species is broad. Taking into account that the anisotropy field of CFO strongly decreases with $\mathrm{T}$, the average reversal fields may be similar at RT and only discernable at low T. It must be kept in mind that an exchange-coupled system with a soft phase particle size close to the critical size ${ }^{10}$ could show an equivalent reversal behavior at $5 \mathrm{~K}$ and RT. Nevertheless, the decrease of remanence at RT for all samples containing FeCo phases allows us to confirm the uncoupling and discard this hypothesis.

In addition, the presence of an uncoupled soft phase with Curie temperature below RT is plausible. Given the coexistence of various Fe-Co oxide and metallic species, a fraction of particles may possess a small enough magnetic volume and the appropriate composition. In that case, at RT, the reversal would occur as a single rotation. However, it is worth noting that this effect alone does not explain the observations, as the increase in $\mathrm{M}_{\mathrm{s}}$ at $\mathrm{RT}$ indicates. In any case, in a system with broad size distributions, we cannot discard the fact that a fraction of particles might be effectively exchange-coupled while others are not. What our results suggest is that the population of uncoupled particles dominates.

In summary, phenomenology associated with exchangespring magnets has been observed in $\mathrm{CFO} / \mathrm{FeCo}$ composites: increase of $M_{r}$ and $M_{s}$; decrease of coercivity; single-phase hysteresis loop at RT. However, exchange-uncoupling between the hard ferrite and the soft metal has been demonstrated to be predominant. The $\mathrm{M}_{\mathrm{r}}$ enhancement is explained in light of the structural and compositional variations induced by the thermal treatments of the CFO particles. Temperature dependence of magnetic parameters may yield single-phase hysteresis loops at RT in uncoupled systems; hence, magnetization curves at low temperature constitute an essential test to establish effective exchange-coupling. We caution that extremely careful and systematic work must be undertaken in order to quantify and optimize the extent of exchange-coupling and energy-product gain that CFO-based composites may provide.

The authors would like to thank financial support from the European Comission through the project NANOPYME FP7-NMP-2012-SMALL-6 NANOPYME (No. 310516), and from Spanish Ministerio de Economía y Competitividad through the Juan de la Cierva program and through Project Nos. MAT2013-48009-C4-1-P, MAT2011-27470-C02-02, and MAT2012-38045-C04-01. Dr. F. Rubio-Marcos is also indebted to CSIC for a "Junta de Ampliación de Estudios", Contract (Ref. JAEDOC071), which is co-financed with FEDER funds. The authors also thank Dr. Dmitry Berkov and Dr. César de Julián for invaluable advice.

${ }^{1}$ L. H. Lewis and F. Jiménez-Villacorta, Metall. Mater. Trans. A 44, 2 (2012).

${ }^{2}$ See http://www.nanopyme-Project.eu/ for further details and references.

${ }^{3}$ J. S. Jiang, J. E. Pearson, Z. Y. Liu, B. Kabius, S. Trasobares, D. J. Miller, S. D. Bader, D. R. Lee, D. Haskel, G. Srajer, and J. P. Liu, Appl. Phys. Lett. 85, 5293 (2004).

${ }^{4}$ H. Zeng, J. Li, J. P. Liu, Z. L. Wang, and S. Sun, Nature 420, 395 (2002).

${ }^{5}$ X. Liu, S. He, J.-M. Qiu, and J.-P. Wang, Appl. Phys. Lett. 98, 222507 (2011).

${ }^{6}$ X. Shen, F. Song, J. Xiang, M. Liu, Y. Zhu, and Y. Wang, J. Am. Ceram. Soc. 95, 3863 (2012).

${ }^{7}$ D. Roy and P. S. A. Kumar, J. Appl. Phys. 106, 073902 (2009).

${ }^{8}$ M. A. Radmanesh and S. A. Seyyed Ebrahimi, J. Magn. Magn. Mater. 324, 3094 (2012).

${ }^{9}$ X. Liu, W. Zhong, B. Gu, and Y. Du, J. Appl. Phys. 92, 1028 (2002).

${ }^{10}$ E. F. Kneller and R. Hawig, IEEE Trans. Magn. 27, 3588 (1991).

${ }^{11}$ Y. Li, Y. Hu, J. Huo, and H. Jiang, Ind. Eng. 51, 11157 (2012).

${ }^{12}$ F. Zan, Y. Ma, Q. Ma, Y. Xu, Z. Dai, G. Zheng, M. Wu, and G. Li, J. Am. Ceram. Soc. 96, 3100 (2013).

${ }^{13}$ F. L. Zan, Y. Q. Ma, Q. Ma, G. H. Zheng, Z. X. Dai, M. Z. Wu, G. Li, Z. Q. Sun, and X. S. Chen, J. Alloys Compd. 553, 79 (2013).

${ }^{14}$ J. Xiang, X. Zhang, J. Li, Y. Chu, and X. Shen, Chem. Phys. Lett. 576, 39 (2013).

${ }^{15}$ N. Viart, R. S. Hassan, C. Mény, P. Panissod, C. Ulhaq-Bouillet, J. L. Loison, G. Versini, F. Huber, and G. Pourroy, Appl. Phys. Lett. 86, 192514 (2005).

${ }^{16}$ J. M. Soares, F. O. Cabral, J. H. de Araujo, and F. L. Machado, Appl. Phys. Lett. 98, 072502 (2011).

${ }^{17}$ D. Roy, K. V. Sreenivasulu, and P. S. Anil Kumar, Appl. Phys. Lett. 103, 222406 (2013).

${ }^{18}$ G. C. P. Leite, E. F. Chagas, R. Pereira, R. J. Prado, A. J. Terezo, M. Alzamora, and E. Baggio-Saitovitch, J. Magn. Magn. Mater. 324, 2711 (2012). 
${ }^{19}$ F. de Assis Olimpio Cabral, F. L. de Araujo Machado, J. H. de Araujo, J. M. Soares, A. R. Rodrigues, and A. Araujo, Magn. IEEE Trans. 44, 4235 (2008). ${ }^{20}$ Y. Zhang, Z. Yang, B. Zhu, S. Chen, X. Yang, R. Xiong, and Y. Liu, J. Alloys Compd. 567, 73 (2013).

${ }^{21}$ A. F. Júnior, V. Zapf, and P. Egan, J. Appl. Phys. 101, 09 M506 (2007).
${ }^{22}$ M. Sivakumar, S. Kanagesan, R. Suresh Babu, S. Jesurani, R. Velmurugan, C. Thirupathi, and T. Kalaivani, J. Mater. Sci. Mater. Electron. 23, 1045 (2012).

${ }^{23}$ E. F. Kneller and F. E. Luborsky, J. Appl. Phys. 34, 656 (1963).

${ }^{24}$ G. Rowlands, J. Phys. D. Appl. Phys. 9, 1267 (1976). 